\title{
A relação da alimentação com o surgimento de doenças oncológicas: avaliação do conhecimento de universitários brasileiros
}

\author{
Relationship between food and onset of oncological diseases: an evaluation of the \\ knowledge of brazilian university students
}

Yasmin Moreira da Silva ${ }^{1}$, Leda Ferraz ${ }^{2}$, Danila Fernanda Rodrigues Frias ${ }^{3}$

\section{RESUMO}

Introdução: A alimentação e a nutrição inadequadas são responsáveis por grande parte dos casos de doenças oncológicas no Brasil. Objetivo: Avaliar o nível de conhecimento de universitários sobre a relação da alimentação com o surgimento de doenças oncológicas. Metodologia: Realizou-se entrevistas com 188 discentes de diversos cursos de graduação. Os dados obtidos foram tabulados e analisados por meio de cálculo de médias simples, números absolutos e porcentagem. Resultados: A ocorrência de câncer em membro da família foi declarada por $58 \%$ e o câncer de mama o mais relatado. Quando questionados sobre a ocorrência de câncer desencadeado ou não por alimentos, $80 \%$ declararam que sim, sendo os alimentos industrializados, os vegetais com uso de agrotóxico e os transgênicos os mais citados. Relacionado a prevenção de câncer por meio de alimentos, $82 \%$ declararam que sim, e citaram frutas, verduras e legumes, $53 \%$ dos entrevistados, consumiam frutas, verduras e legumes semanalmente, $80 \%$ carne, ovos, leite e derivados; $80 \%$ açucares e gorduras; $58 \%$ embutidos; e $80 \%$ bebida industrializada. Apenas $42,5 \%$ dos entrevistados relataram já ter procurado auxílio de um nutricionista. Conclusões: Notou-se a necessidade de conscientizar a população dos perigos da alimentação e fornecer informações para contribuir com a prática da alimentação saudável.

Palavras-chave: Conscientização. Dieta saudável. Neoplasias. Prevenção

\section{ABSTRACT}

Introduction: Inadequate eating habits and nutrition are responsible for most cases of oncological diseases in Brazil. Objective: Undertaken to assess the level of knowledge of university students about the relationship between food and the onset of oncological diseases. Methods: Interviews were conducted with 188 students from undergraduate courses. The obtained data were tabulated and analyzed by calculating simple averages, absolute numbers and percentage. Results: Occurrence of cancer in the member of the family was declared by $58 \%$, with breast cancer being the most reported. When asked about the occurrence of cancer being triggered by food, $80 \%$ stated "yes", among industrialized foods; vegetables grown with the use of pesticides and transgenics most cited. As regards cancer prevention through food, $82 \%$ of the respondents were positive, citing fruits and vegetables. fifty-three percent $(53 \%)$ stated they consumed fruits and vegetables; $80 \%$ meat, eggs, milk and dairy products; $80 \%$ sugars and fats; $58 \%$ sausages/stuffed meat products; and $80 \%$ industrialized beverage. Only $42.5 \%$ of respondents reported having sought the aid of a nutritionist. Conclusions: The results point to a need to raise awareness in the population about the dangers associated with food habits and to provide information that contributes to the adoption of healthy eating.

Keywords: Awareness. Healthy diet. Neoplasms. Prevention.
Graduação em Nutrição, Universidade Brasil, Campus Fernandópolis.

E-mail:

yasmin.silva63@etec.sp.gov.br

2 Doutora, Docente do curso de Nutrição, Universidade Brasil, Campus Fernandópolis.

E-mail:

leda.silva@universidadebrasil.ed u.br

Doutora, Docente titular do Programa de Mestrado em Ciências Ambientais, Universidade Brasil, Campus Fernandópolis

E-mail:

danila.frias@universidadebrasil.e du.br 


\section{INTRODUÇÃOO}

O aumento gradativo da ocorrência de neoplasias no mundo todo vem ocorrendo a cada dia. Acredita-se, que o processo de industrialização, produção e comercialização que utilizam, na maioria das vezes, produtos químicos para aumentar a vida útil dos alimentos, seja o principal fator relacionado com o aumento da casuística desta enfermidade (INSTITUTO ONCOGUIA, 2020).

O organismo humano está em constante desenvolvimento, pois as células crescem e se dividem, para efetuar a manutenção e reparos constantes dos tecidos que o formam. Para a manutenção destas células, o consumo de alimentos é essencial, e estes interferem diretamente no metabolismo celular (OPPERMANN, 2014).

O câncer surge quando os mecanismos que regulam o comportamento natural do organismo se alteram. $O$ desenvolvimento habitual das células como seu crescimento, proliferação, diferenciação e sobrevida, caminham a favor da regularização e da satisfação das necessidades do organismo como um todo. Esta regularização se perde nas células neoplásicas, fazendo com que estas cresçam e se multipliquem de maneira descontrolada (FIGUEIREDO, 2013).

Uma vez iniciada, esta multiplicação desordenada torna-se agressiva e pode se espalhar para diferentes regiões do organismo, o que irá interferir diretamente nas funções dos tecidos e órgãos devido a formação de tumores (WEINBERG, 2008). Os diversos tipos de câncer diferem-se conforme o local invadido. Quando atingem os tecidos epiteliais são chamados de carcinomas, caso ocorra nos tecidos conjuntivos sua nomenclatura é sarcoma (INSTITUTO ONCOGUIA, 2020).

A alimentação inadequada é responsável por cerca de $20 \%$ dos casos de doenças oncológicas em nosso país, pois existem substâncias presentes nos alimentos (agrotóxicos, conservantes, gorduras) que atuam em desfavor à célula, causando este crescimento desordenado (WEINBERG, 2008). Esta prevalência seria facilmente diminuída com a utilização de uma alimentação rica em frutas, legumes, verduras, e pobre em alimentos processados, como os prontos para consumo e bebidas açucaradas (GAROFALO, et al., 2005; FIGUEIREDO \& SILVA, 2008).

Com a incorporação de uma alimentação saudável e a prática regular de atividade física, aproximadamente um em cada três casos dos tipos de câncer mais prevalentes poderiam ser evitados (PRADO, 2014). Mas, segundo dados do Instituto Brasileiro de 
Geografia e Estatística (IBGE), 9 em cada 10 brasileiros não ingerem frutas, verduras e legumes diariamente (INCA, 2020).

O desconhecimento da população em relação a ações preventivas relacionadas ao aparecimento de doenças oncológicas é evidente, mostrando assim, a importância da implementação de ações educativas que visem disseminar informações e conscientizar a população com relação a prática da alimentação saudável com intuito de promover e proteger a saúde. Por este motivo, o presente trabalho teve por objetivo avaliar o conhecimento de universitários sobre a relação da alimentação com o surgimento de doenças oncológicas.

\section{MATERIAL E METODOS}

O projeto foi desenvolvido na Universidade Brasil, Campus de Fernandópolis, SP. O estudo caracterizou-se por ser uma pesquisa quantitativa, descritiva e exploratória, composta por 188 estudantes da referida instituição de ensino, não contemplando discentes de cursos da área de saúde devido ao conhecimento que estes poderiam ter adquirido durante a graduação, o que induziria a resposta de uma prática alimentar correta.

O cálculo amostral foi realizado pelo método de população finita, sendo utilizado:

Nível de confiança: $95 \%$

Quantidade de acerto esperado: $50 \%$

Quantidade de erro esperado: $50 \%$

População total: 4500 alunos

Nível de precisão: $7 \%$

Foi desenvolvido um questionário estruturado com questões relacionadas a rotina alimentar dos entrevistados e seu nível de conhecimento sobre alimentos cancerígenos. Os alunos foram abordados de forma casual no pátio da Universidade, e em seguida o entrevistador fez esclarecimentos sobre a pesquisa e realizou o convite para participação. Após o aceite, foi aplicado o questionário, por meio de entrevista. A pesquisa foi efetuada após a aprovação pelo Comitê de Ética em Pesquisa da Universidade Brasil, com número de protocolo 2.082.376.

Com a realização do diagnóstico de situação foi possível formar um banco de dados após a digitalização das informações coletadas em planilhas do software Microsoft Office Exce ${ }^{\oplus}$. Em seguida, os mesmos foram analisados por meio de estatística descritiva 
(cálculo de médias simples, número absoluto e porcentagem). Os resultados foram dispostos em forma de tabelas e gráficos.

\section{RESULTADOS}

Dentre os entrevistados, 19 eram do curso de agronomia, 23 arquitetura, 58 direito, 38 engenharia química, 25 engenharia civil e 25 pedagogia. A maioria $(33,5 \%)$ cursavam o $4^{\circ}$ semestre, $59,6 \%$ eram do sexo feminino, e com idade variando de 18 a 69 anos conforme descrito na Tabela 1.

\begin{tabular}{|c|c|c|c|}
\hline VARIÁVEL & CATEGORIA & QUANTIDADE & PERCENTUAL \\
\hline \multirow[t]{6}{*}{ Curso } & Agronomia & 19 & $10,1 \%$ \\
\hline & Arquitetura & 23 & $12,2 \%$ \\
\hline & Direito & 58 & $30,9 \%$ \\
\hline & Engenharia Química & 38 & $20,2 \%$ \\
\hline & Engenharia Civil & 25 & $13,3 \%$ \\
\hline & Pedagogia & 25 & $13,3 \%$ \\
\hline \multirow[t]{4}{*}{ Semestre } & $2^{0}$ & 56 & $29,8 \%$ \\
\hline & $3^{\circ}$ & 33 & $17,6 \%$ \\
\hline & $4^{\circ}$ & 63 & $33,5 \%$ \\
\hline & $6^{0}$ & 36 & $19,1 \%$ \\
\hline \multirow[t]{2}{*}{ Sexo } & Feminino & 112 & $59,6 \%$ \\
\hline & Masculino & 76 & $40,4 \%$ \\
\hline \multirow[t]{4}{*}{ Idade } & $18-20$ anos & 110 & $58,5 \%$ \\
\hline & $21-25$ anos & 42 & $22,4 \%$ \\
\hline & $26-30$ anos & 17 & $9 \%$ \\
\hline & Acima de 30 anos & 19 & $10,1 \%$ \\
\hline
\end{tabular}

Fonte: Elaborada pelos autores

Quando contestados sobre quem compra os alimentos em sua casa, o resultado está expresso na Tabela 2.

Tabela 2. Responsável pela aquisição dos alimentos que são consumidos pelos discentes entrevistados na Universidade Brasil, Campus Fernandópolis, 2019.

RESPONSÁVEL PELA COMPRA DE ALIMENTOS ENTREVISTADO

PAI/PADRASTO

MÃE/MADRASTA

TIO/TIA

MARIDO/ESPOSA

AVÓ/MÃE

ENTREVISTADO/MÃE

IRMÃO/IRMÃ

$\mathrm{PAI} / \mathrm{MÃE}$

TODOS OS RESIDENTES
TOTAL

$\begin{array}{cc}52 & 27,6 \% \\ 9 & 4,8 \% \\ 79 & 42 \% \\ 2 & 1 \% \\ 9 & 4,8 \% \\ 4 & 2,2 \% \\ 6 & 3,2 \% \\ 3 & 1,6 \% \\ 13 & 6,9 \% \\ 11 & 5,9 \%\end{array}$

Fonte: Elaborada pelos autores

A população que reside com estes discentes compreendeu 59 crianças ( 0 a 12 anos), 57 adolescentes (13 a 18 anos), 554 adultos (19 a 59 anos), e 27 idosos (60 anos 
ou mais), totalizando 697 indivíduos. A ocorrência de câncer na residência foi declarada por $15,5 \%$ dos entrevistados, sendo o câncer de pele o mais relatado (Figura 1).

Figura 1. Tipo de câncer ocorrido em familiares que residem na mesma casa relatado pelos discentes entrevistados na Universidade Brasil, Campus Fernandópolis, 2019.

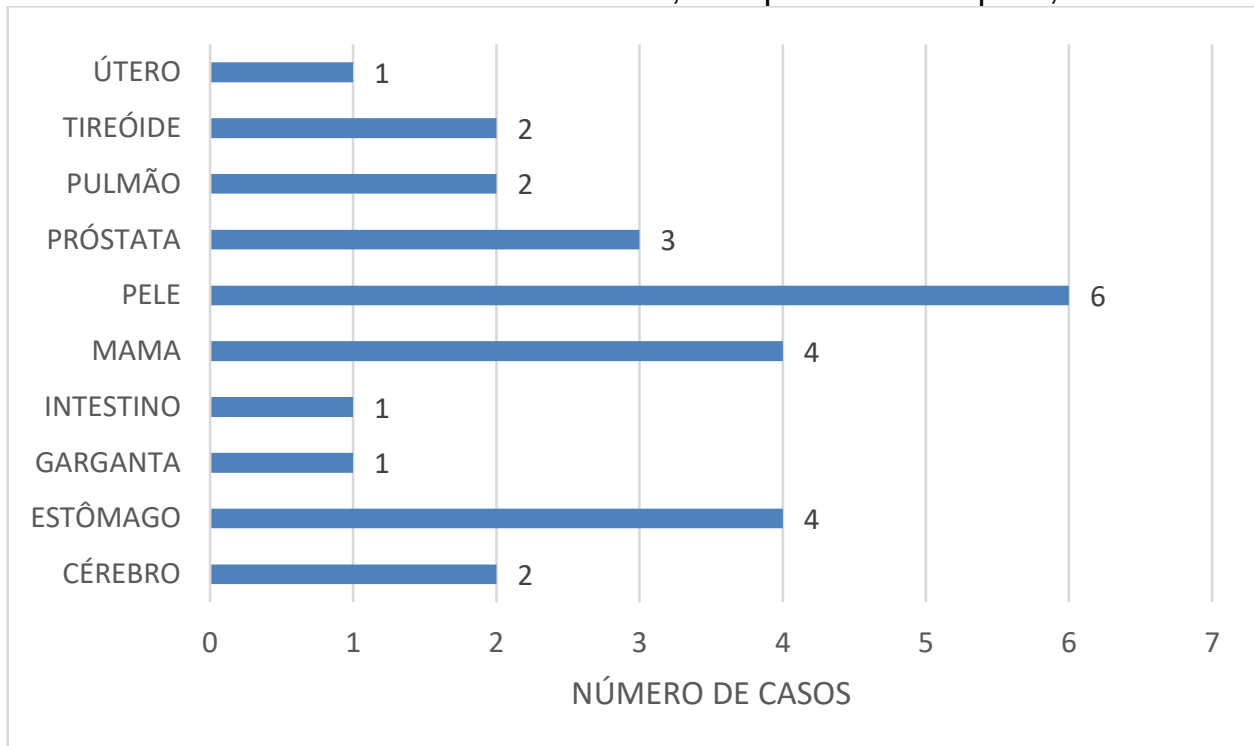

Fonte: Elaborada pelos autores

Quando o questionamento se referiu a casos de câncer na família, 58\% dos entrevistados declararam sofrer com este problema, sendo o câncer de mama o de maior ocorrência (Figura 2).

Figura 2. Tipo de câncer em familiares relatado pelos discentes entrevistados na Universidade Brasil, Campus Fernandópolis, 2019.

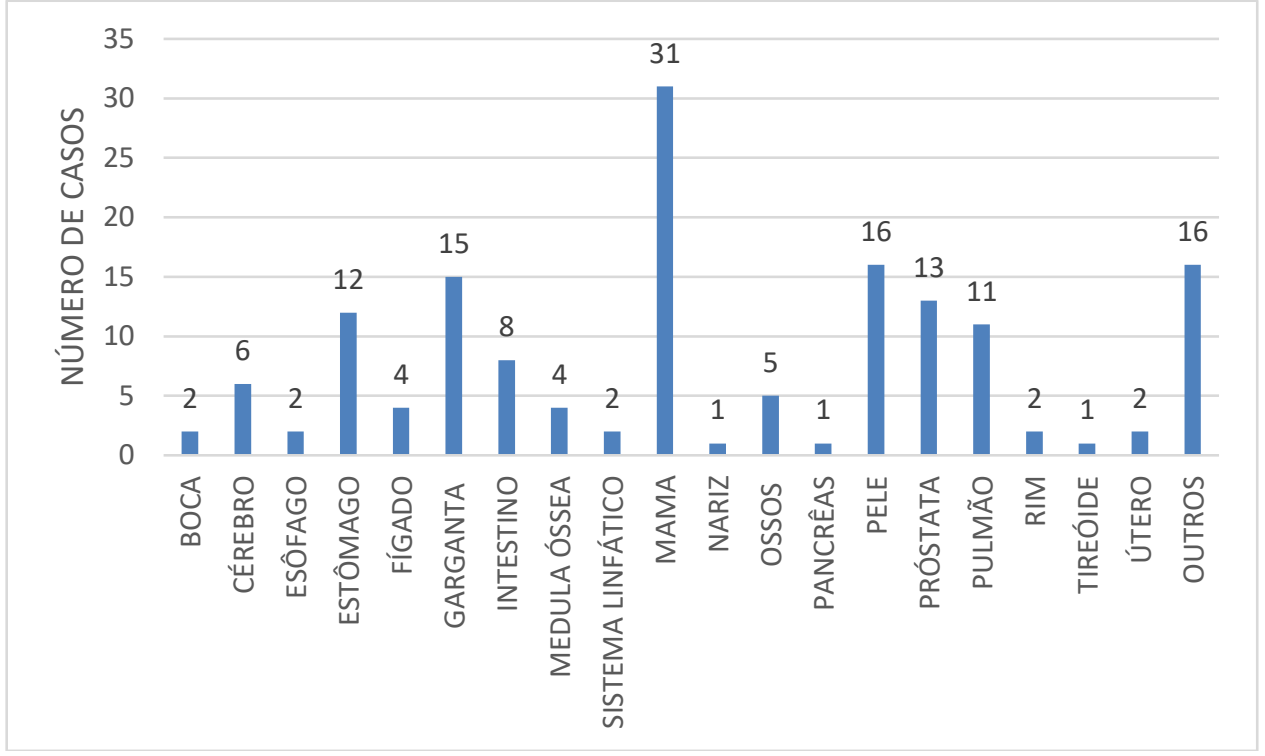

Fonte: Elaborada pelos autores

Quando questionados sobre a ocorrência de câncer desencadeada ou não por alimentos, $80 \%$ dos universitários declararam que sim, os alimentos podem causar 
câncer. Dentre os alimentos mais citados como possíveis causadores estão os industrializados, os vegetais com uso de agrotóxico e os embutidos (Figura 3).

Figura 3. Alimentos que aumentam risco de desenvolvimento de câncer citados pelos discentes entrevistados na Universidade Brasil, Campus Fernandópolis, 2019.

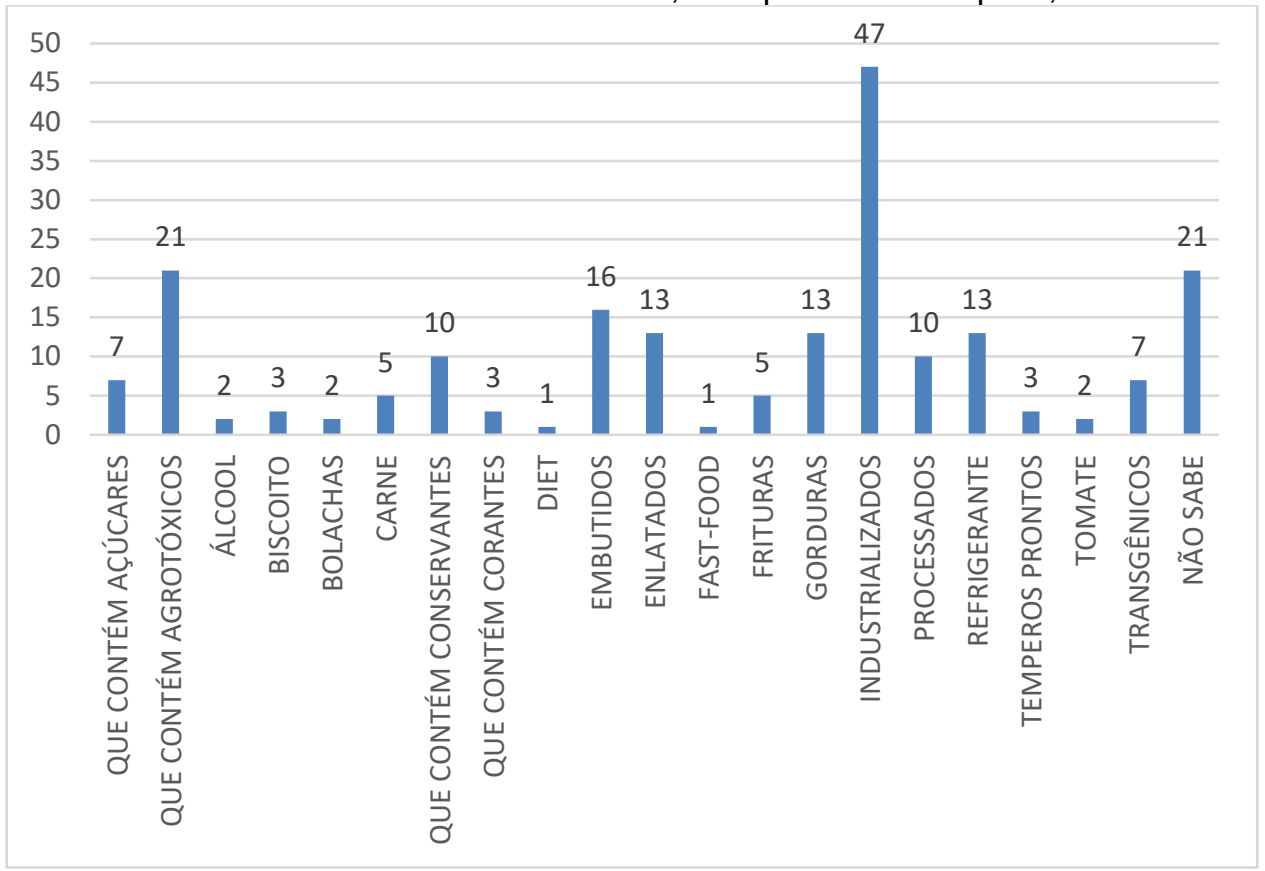

Fonte: Elaborada pelos autores

Nessa pesquisa, $82 \%$ dos discentes relataram que a prevenção das doenças oncológicas pode ser realizada por meio da melhoria na alimentação com o consumo de frutas, verduras e legumes, sendo estes os principais alimentos para prevenção desta enfermidade.

Um fato importante a salientar está relacionado aos hábitos alimentares destes estudantes. $O$ consumo alimentar semanal relatado pelos discentes consta na Tabela 3.

Tabela 3. Alimentos consumidos semanalmente pelos discentes entrevistados na Universidade Brasil,

\begin{tabular}{|c|c|c|}
\hline ALIMENTO & TOTAL & PERCENTUAL \\
\hline FRUTAS & 148 & $78,7 \%$ \\
\hline LEGUMES & 144 & $76,6 \%$ \\
\hline HORTALIÇAS & 125 & $66,5 \%$ \\
\hline CARNES VERMELHAS E OVOS & 177 & $94,2 \%$ \\
\hline LEITE E DERIVADOS & 159 & $84,6 \%$ \\
\hline AÇÚCARES & 169 & $89,9 \%$ \\
\hline GORDURAS E ÓLEOS & 157 & $83,5 \%$ \\
\hline EMBUTIDOS & 109 & $58 \%$ \\
\hline BEBIDAS INDUSTRIALIZADAS & 150 & $79,8 \%$ \\
\hline $\begin{array}{l}\text { OUTROS PRODUTOS } \\
\text { INDUSTRIALIZADOS }\end{array}$ & 85 & $45,2 \%$ \\
\hline
\end{tabular}

Fonte: Elaborada pelos autores 
Nota-se na Tabela 3 o elevado consumo de produtos considerados com potencial cancerígeno, principalmente, açúcares, gorduras e óleos, embutidos, bebidas industrializadas e outros produtos industrializados. $\mathrm{O}$ consumo de bebidas industrializadas semanalmente foi relatado por $79,8 \%$ dos estudantes.

Quando indagados sobre o favorecimento do aparecimento de câncer por meio do consumo de alimentos industrializados, $91 \%$ dos entrevistados afirmaram esta possibilidade. Mesmo conscientes, $76,6 \%$ afirmaram consumir semanalmente produtos considerados de risco para o desenvolvimento da doença.

O consumo semanal de frutas, verduras e hortaliças se restringiu a $74 \%$ dos participantes da pesquisa.

\section{DISCUSSÃO}

O sexo feminino teve predomínio nesta pesquisa assim como na de Busato et al. (2015), Aquino; Pereira; Reis (2015), Marconato; Silva; Frasson (2016), Macedo et al. (2019) e Santos et al. (2019). Segundo o censo realizado em 2018, a maioria dos estudantes universitários são do sexo feminino (BRASIL, 2019), o que poderia justificar o resultado deste trabalho.

A média de idade encontrada nessa pesquisa foi de 23 anos. Dado este que corrobora com a pesquisa de Aquino; Pereira; Reis (2015), Marconato; Silva; Frasson (2016), Oliveira et al. (2017) e Santos et al. (2019). Geralmente a maioria das pessoas nesta idade encontra-se em período universitário e já faz suas escolhas alimentares que podem refletir positiva ou negativamente em sua saúde pelo resto de sua vida.

A Tabela 2 revela, que na maioria dos lares dos discentes entrevistados, a aquisição dos alimentos é realizada pela mãe/madrasta (42\%), seguido pelos próprios entrevistados $(27,6 \%)$. A presença da família para escolha dos alimentos e preparo dos mesmos está diretamente relacionada com a percepção alimentar de cada um (BUSATO et al., 2015). Desta forma, vê-se a necessidade de intensificação nas medidas de instrução aos universitários e a seus familiares com relação a prática de alimentação saudável.

A alimentação inadequada é responsável por cerca de $20 \%$ dos casos de câncer no mundo. A estimativa no Brasil para o ano de 2020 é de 625.370 casos novos de câncer no pais. Dentre os tipos de câncer mais prevalentes no Brasil, destacam-se nos homens o de próstata, pulmão e intestino, e nas mulheres os de mama, intestino e útero. 
Já no estado de São Paulo, os dados são semelhantes aos encontrados no Brasil, porém o terceiro de maior ocorrência entre as mulheres é o câncer de tireóide (INCA, 2020).

Alimentos industrializados e carnes processadas possuem alto risco para desenvolvimento de doenças oncológicas. Carnes vermelhas devem ser consumidas com moderação e o cuidado com o preparo do alimento também deve ser analisado, pois frituras e churrasco podem alterar a composição do alimento tornando assim um fator de risco para o desenvolvimento da doença (MUNHOZ et al., 2016).

O equilíbrio alimentar, aliado a uma dieta balanceada e rica em fibras, vitaminas e minerais, favorece a prevenção ao câncer, e auxilia também no tratamento do estado clínico já iniciado (FIGUEIREDO; SILVA, 2008). Nessa pesquisa, $82 \%$ dos discentes relataram que a prevenção das doenças oncológicas pode ser realizada por meio da melhoria na alimentação com o consumo de frutas, verduras e legumes, sendo estes os principais alimentos para prevenção desta enfermidade.

Alimentos que possuem betacaroteno, flavonoides, vitamina $\mathrm{C}$, fibras e selênio, como as frutas, verduras, legumes, alimentos integrais e oleoginosas, são responsáveis pela prevenção do câncer, como afirmaram Scwarcz; Berkoff (2006), Mahan; EscottStump; Krause (2010) e Oliveira (2015).

A Tabela 3 demonstra o elevado consumo de produtos considerados com potencial cancerígeno, principalmente, açúcares, gorduras e óleos, embutidos, bebidas industrializadas e outros produtos industrializados.

Este fato pode estar relacionado a globalização que ocorreu ao longo das últimas décadas que apresentou ao mundo ideias de alimentos de preparo mais rápido; a falta de tempo disponível devido ao excesso de trabalho e estudos; e a mudança da casa dos pais. Estes fatores podem ser os responsáveis por impedir a adoção de estilos de vida mais saudáveis, o que acaba impulsionando a escolhas de comidas de preparo mais rápido e a diminuição da prática de atividades físicas (OLIVEIRA et al., 2016; SANTOS et al., 2019).

O consumo de bebidas industrializadas semanalmente foi relatado por $79,8 \%$ dos estudantes. Dado este bastante alarmante quando comparado a outros estudos, como por exemplo o realizado por Silva et al. (2019) que citaram consumo de deste tipo de bebida por apenas $23,4 \%$ dos universitários entrevistados. Já Macedo et al. (2019) constatou o consumo de refrigerantes por $61,4 \%$. O consumo de bebidas adoçadas pode levar ao excesso de peso e ao desenvolvimento de câncer (IARC, 2018). 
A maioria dos entrevistados relataram saber sobre o favorecimento do aparecimento de câncer por meio do consumo de alimentos industrializados, mas mesmo assim consumiam de forma elevada. Estes alimentos geralmente possuem na sua composição substâncias utilizadas para conservação e realçar o sabor, como nitratos e nitritos, que são transformados em nitrosamina no trato gastrointestinal. A nitrosamina é uma das principais substâncias com potencial cancerígeno (HUWE et al., 2014).

Outras pesquisas também revelaram elevado consumo de alimentos pouco saudáveis, como balas, doces, chocolates, salgados fritos, refrigerantes, hambúrgueres e embutidos (OLIVEIRA et al., 2016; ALMEIDA et al., 2017; MACEDO et al., 2019; SILVA et al., 2019).

O desregramento alimentar pode causar com o passar do tempo transtornos e patologias preocupantes ao organismo, assim como, a alta ingestão de gorduras, embutidos e produtos industrializados podem estimular o surgimento do estágio inicial neoplásico (FIGUEIREDO; SILVA, 2008).

O consumo de alimentos com potencial carcinogênico está aumentando entre os universitários, por serem de fácil e prático preparo, o que torna uma situação bastante preocupante devido ao risco de desenvolvimento de câncer neste extrato da população (OLIVEIRA et al., 2014).

O consumo semanal de frutas, verduras e hortaliças foi relatado por $74 \%$ dos entrevistados. Pesquisa realizada por Oliveira et al. (2016) demonstrou que $14 \%$ dos jovens não consumiam frutas e $10 \%$ não consumiam verduras, valor menor que o encontrado nesta pesquisa, que revelou o não consumo de frutas, verduras e hortaliças por $26 \%$ dos entrevistados. O dado encontrado nesse estudo evidenciou o inadequado consumo de frutas e verduras, visto que o preconizado pelo Ministério da Saúde é de no mínimo 3 porções ao dia destes alimentos (BRASIL, 2008).

Almeida et al. (2017) realizaram um estudo com universitários e constataram que verduras eram consumidas diariamente por $54 \%$ dos entrevistados e frutas por $45 \%$, assim como Macedo et al. (2019) relataram o consumo semanal de verduras e legumes por praticamente $100 \%$ dos entrevistados. Já Silva et al. (2019) constataram em sua pesquisa que a minoria dos universitários consumia hortaliças e verduras diariamente, corroborando com este estudo.

O consumo de uma dieta rica em frutas, verduras e hortaliças é fundamental para prevenção de doenças oncológicas e da obesidade (HYPPOLITO; RIBEIRO, 2014). A alimentação saudável promove proteção contra o câncer, ou seja, uma alimentação rica 
em alimentos de fonte vegetal e pobre em alimentos ultra processados pode prevenir cerca de 1/3 dos cânceres existentes (INCA, 2019).

De acordo com Figueiredo; Silva (2008), mesmo que existam diversos fatores que originam a carcinogênese, a alimentação vem tomando destaque, seja ela como retardadora ou intensificadora, dessa forma, seu papel é de extrema relevância para o surgimento da doença. Mesmo assim, nessa pesquisa, apenas $42,5 \%$ dos entrevistados relataram já ter procurado auxílio de um nutricionista.

As questões culturais estão bastante relacionadas a baixa procura de apoio de nutricionista. Além disso, os fatores econômicos também influem na ingestão de alimentos saudáveis, pois estudos demonstraram que dietas com alto teor de frutas e vegetais são mais caras que as outras dietas, por isso, as restrições econômicas de muitas famílias induz ao consumo de produtos mais baratos que geralmente são alimentos com alto teor de energia, a base de óleos e açúcares (DEFANTE; NASCIMENTO; LIMA-FILHO, 2015; MOTTER et al., 2015; OLIVEIRA et al., 2016).

Quando o discente inicia seus estudos na universidade, muitos saem da casa de seus pais, e acabam tornando-se responsáveis pela gestão, desde financeira até alimentar, de seu novo lar. Dessa forma, é bastante complexo ao universitário conseguir conciliar as atividades acadêmicas com a nova vida socioambiental que passou a usufruir, portanto o consumo de refeições pré-preparadas, lanches, fast food, doces, produtos industrializados é justificado devido à falta de tempo e dinheiro, além de que, não é comum, dentro do ambiente universitário, a oferta de alimentos saudáveis e com custo baixo (PIRES et al., 2013; MIRANDA et al., 2014).

\section{CONSIDERAÇÖES FINAIS}

Após o desenvolvimento dessa pesquisa, concluiu-se que os universitários e seus familiares possuem grande defasagem quando se trata do assunto alimentação saudável. E visto que, há várias evidências da importância da alimentação relacionadas ao índice de desenvolvimento de doenças oncológicas, notou-se a necessidade de investimento em ações que visem a promoção da saúde dentro das Instituições de Ensino Superior extrapolando também para a conscientização da população sobre perigos da alimentação inadequada visando diminuir a incidência das doenças oncológicas.

Esse plano de ação deve ser realizado por meio do fornecimento de subsídios que contribuam com a prática da alimentação saudável para promoção da saúde. Além da 
diminuição da ocorrência de câncer, os efeitos de uma dieta saudável acompanhada de exercícios físicos contribuirão também para a diminuição de outras doenças não transmissíveis como o diabetes tipo II, hipertensão arterial e doenças cardiovasculares.

\section{REFERENCIAS}

ALMEIDA, L., et al. Alimentação como fator de risco para câncer de intestino em Universitários. Revista Brasileira de Promoção à Saúde, v.30, n.1, p.72-78, 2017.

AQUINO, J.K.; PEREIRA, P.; REIS, V.M.C.P. Hábito e consumo alimentar de estudantes do curso de Nutrição das faculdades de Montes Claros-Minas Gerais. Revista Multitexto, v.3, n.1, p.82-88, 2015.

BRASIL. Instituto Nacional de Estudos e Pesquisas Educacionais Anísio Teixeira (Inep). Censo da Educação Superior 2018. 2019. Disponível em:

http://download.inep.gov.br/educacao_superior/censo_superior/docum entos/2019/censo_da_educacao_superior_2018-notas_estatisticas.pdf. Acesso em: 19 mar. 2020.

BRASIL. Ministério da Saúde. Secretaria de Atenção à Saúde. Guia alimentar para a população brasileira: promovendo a alimentação saudável. Brasília: Ministério da Saúde, 2008, $210 \mathrm{p}$.

BUSATO, M.A., et al. Ambiente e alimentação saudável: Percepções e práticas de estudantes universitários. Semina Ciências Biológicas e da Saúde, v.36, n.2, p.75-84, 2015.

DEFANTE, L.R.; NASCIMENTO, L.D.O.; LIMA-FILHO, D.O. Comportamento de consumo de alimentos de famílias de baixa renda de pequenas cidades brasileiras: o caso de Mato Grosso do Sul. Interações, v.16, n.2, p.265-276, 2015.

FIGUEREDO, V.; SILVA, C.H.C. A influência da alimentação como agente precursor, preventivo e redutor do câncer. Universitas Ciências da Saúde, v.1, n.2, p.317-325, 2008.

GARÓFOLO, A. et al. Dieta e câncer: um enfoque epidemiológico. Revista de Nutrição, v.17, n.4, p.491-505, 2004.

HUWE, F.G., et al. Avaliação das características clínicas e epidemiológicas e sobrevida global de pacientes portadores de câncer colorretal. Revista Epidemiologia e Controle da Infecção, v.3, n.4, p.112-116, 2014.

HYPPOLITO, K.P.P.; RIBEIRO, K.A.R. Importância da nutrição na prevenção e no tratamento de neoplasias. Interciência \& Sociedade, v.3, n.2, p.51-59, 2014.

INCA. Instituto Nacional de Câncer José Alencar Gomes da Silva. O que é câncer? 2020. [acesso em 20 mar. 2020]. Disponível em: https://www.inca.gov.br/numeros-de-cancer. 
INSTITUTO ONCOGUIA. Estimativas de câncer no mundo. 2015. Disponível em: http://www.oncoguia.org.br/conteudo/estimativas-no-mundo/ 1706/1/. Acesso em 24 jan. 2020.

MACEDO, T.T.S., et al. Perfil alimentar, clínico e padrão de atividade física em ingressantes universitários de enfermagem. Revista Cubana de Enfermería, v.35, n.1, p.1-14, 2019.

MAHAN, L.K.; ESCOTT-STUMP, S. Krause: Alimentos, Nutrição e Dietoterapia. 12ed. São Paulo: Editora ELSEVIER, 2010, 1358p.

MARCONATO, M.S.F.; SILVA, G.M.M.; FRASSON, T.Z. Habito alimentar de universitários iniciantes e concluintes do curso de nutrição de uma universidade do interior paulista.

Revista Brasileira de Obesidade, Nutrição e Emagrecimento, v.10, n.58, p.180-188, 2016.

MIRANDA, M.L.P., et al. Avaliação antropométrica e análise do consumo alimentar de universitários da Faculdade de Ciências Aplicadas-UNICAMP. Segurança Alimentar e Nutricional, v.21, n.2, p.461-468, 2014.

MOTTER, A.F., et al. Pontos de venda de alimentos e associação com sobrepeso/obesidade em escolares de Florianópolis, Santa Catarina, Brasil. Cadernos de Saúde Pública, v.31, n.3, p.620-632, 2015.

MUNHOZ MP., et al. Efeito do exercício físico e da nutrição na prevenção do câncer. Revista de Odontologia, v.37, n.2, p.9-16, 2016.

OLIVEIRA MC., et al. Ambientes alimentares universitários: Percepções de estudantes de Nutrição de uma instituição de ensino superior. Demetra, v.12, n.2, p.431-445, 2017.

OLIVEIRA, J.S., et al. ERICA: uso de telas e consumo de refeições e petiscos por adolescentes brasileiros. Revista de Saúde Pública, v.50, n.1, p.1-9, 2016.

OLIVEIRA, V.A., et al. Relação entre consumo alimentar da população nordestina e alto índice de câncer gástrico nesta região. Revista Intertox, v.7, n.3, p.6-24, 2014.

OPPERMANN, C. Entendendo o Câncer. São Paulo: Artmed; 2014. 95 p.

PIRES, C.G.S., et al. Prática de atividade física entre estudantes de graduação em enfermagem. Acta Paulista de Enfermagem, v.26, n.5, p.436-443, 2013.

PRADO, B. Influência dos hábitos de vida no desenvolvimento do câncer. Ciência e Cultura, v.66, n.1, p.21-24, 2014.

SANTOS, A.C.F., et al. Consumo de alimentos protetores e risco para desenvolvimento de câncer entre estudantes da saúde. Demetra, v.14, n.1, p.1-21, 2019.

SCWARCZ, J.; BERKOFF, F. Alimentos saudáveis, alimentos perigosos: guia prático para uma alimentação rica e saudável. Rio de Janeiro: Reader's Digest, 2006, 400p.

SILVA, H.S., et al. Consumo de fibras alimentares por universitários de Várzea Grande Mato Grosso. Journal Health Biology Science, v.7, n.3, p.248-252, 2019.

WEINBERG, R. A biologia do Câncer. São Paulo: Artmed; 2008, 822 p. 